\title{
A Simultaneous GBT/Fermi Study of Crab Giant Pulses
}

\section{A. V. Bilous ${ }^{a}$, V. I. Kondratiev ${ }^{* b}$, M. A. McLaughlin ${ }^{c}$, S. M. Ransom ${ }^{d}$, M. Lyutikov ${ }^{e}$,} M. Mickaliger ${ }^{c}$, B. Stappers ${ }^{f}$, G. I. Langston ${ }^{g}$

${ }^{a}$ Department of Astronomy, University of Virginia, PO Box 400325, Charlottesville, VA 22904, US

${ }^{b}$ Netherlands Institute for Radio Astronomy (ASTRON), Postbus 2, 7990 AA Dwingeloo, NL

${ }^{c}$ Department of Physics, West Virginia University, Morgantown, WV 26506, US

${ }^{d}$ National Radio Astronomy Observatory, Charlottesville, VA 22903, US

${ }^{e}$ Department of Physics, Purdue University, 525 Northwestern Avenue, West Lafayette, IN 47907-2036, US

${ }^{f}$ Jodrell Bank Centre for Astrophysics, School of Physics and Astronomy, The University of Manchester, Manchester M13 9PL, UK

${ }^{g}$ National Radio Astronomy Observatory, Green Bank, WV 24944, US

E-mail: avb3k@virginia.edu, kondratiev@astron.nl, maura.mclaughlin@mail.wvu.edu, sransom@nrao.edu, lyutikov@purdue.edu, mmickali@mix.wvu.edu, Ben.Stappers@manchester.ac.uk, glangsto@nrao.edu

To constrain the giant pulse (GP) emission mechanism, we have carried out a campaign of simultaneous observations of the Crab pulsar at $\gamma$-ray (Fermi) and radio (Green Bank Telescope) wavelengths. Over 10 hours of simultaneous observations we obtained a sample of 210000 giant pulses (most of them from interpulse (IP) phase window), observed at a radio frequency of 8.9 GHz, and 77 Fermi photons, with energies between $100 \mathrm{MeV}$ and $5 \mathrm{GeV}$. No change in GP generation rate was found on a time scale from 10 to $120 \mathrm{~s}$ around $\gamma$-ray photons and with any possible lag within \pm 40 min between GPs and $\gamma$-ray photons. With $95 \%$ probability the high energy flux in $30 \mathrm{~ms}$ window around giant pulse is no more than 6 times the average pulsed flux. This suggests that giant pulses, at least high-frequency IP GPs, are due to changes in coherence rather than an overall increase in the magnetospheric particle density.

ISKAF2010 Astronomy Meeting, "A New Golden Age for Radio Astronomy”

June 10-14, 2010

Resort Hof van Saksen, Assen, The Netherlands

\footnotetext{
${ }^{*}$ Speaker.
} 


\section{Introduction}

Giant pulses (GPs) are rare phenomenon of sporadic increase of pulsar's radio flux, known for only handful of pulsars. They last from few nanoseconds to a few microseconds, and are clearly a special form of pulsar radio emission [4, 9]. GPs generally occur only in certain narrow ranges of pulse phase that are often coincident with pulses seen at X-ray and $\gamma$-ray energies [5]. Similar to other sporadic variability phenomena seen in pulsar radio emission, GPs could be due to changes in the coherence of the radio emission, variations in the pair creation rate in the magnetosphere, or changes in the beaming direction. Last two possible explanations imply correlation of the radio GPs with the high-energy photons.

Previous studies of Crab pulsar showed that during GPs recorded at about $1 \mathrm{GHz}$ the highenergy flux increases no more than 2.5 times for $50-220 \mathrm{keV}$ photons and 4.6 times for photons with $E>50 \mathrm{MeV}[6,10]$. Also, in the same radio frequency range, there was found a weak radio-optical correlation, namely that optical pulses coincident with radio GPs were on average 3\% brighter than others [12]. Thus, for lower-frequency GPs, the giant pulses emission mechanism, whichever its nature, can include relatively small variations in magnetospheric particle density or beaming direction alteration.

The Fermi Large Area Telescope (LAT), with its large effective area of $\gtrsim 8000 \mathrm{~cm}^{2}$, broad field of view of $2 \mathrm{sr}$, and superior angular resolution of $<3.5^{\circ}$ is a perfect tool for investigating the possible correlation between GPs and $\gamma$-ray photons. For the radio part, using the Green Bank Telescope (GBT) allows one to record a huge number of GPs within a reasonable observing time at frequencies above $4 \mathrm{GHz}$, where GPs are less frequent and might be generated by different physical processes than those below $4 \mathrm{GHz}$ [7]. Here we present the results of simultaneous GBT/LAT observations of the Crab pulsar.

\section{Observations}

The radio observations were carried out in September-October 2009 with the 100-m Robert C. Byrd Green Bank Telescope (GBT) using the new Green Bank Ultimate Pulsar Processor (GUPPI) at a frequency of $8.9 \mathrm{GHz}$. The total bandwidth of $800 \mathrm{MHz}$ was split into 256 frequency channels, and the total intensity was recorded with the sampling interval 2.56-3.84 $\mu \mathrm{s}$. The raw data from every session were dedispersed with the current DM of the Crab pulsar (from Jodrell Bank Crab pulsar monthly ephemeris), using the PRESTO package, and searched for all the single-pulse events with $\mathrm{S} / \mathrm{N}>7$. The lists of events were put into TEMPO2 format and converted to barycentric reference frame. Times of arrival (TOAs) were corrected for delay due to propagation in the ISM. Estimated timing errors due to inaccuracy in DM are less than our time resolution. The system equivalent flux density (SEFD) were mostly determined by the Crab nebula. Flux densities for the Crab nebula were calculated with the relation $S(f)=955 \times(f / \mathrm{GHz})^{-0.27}$ Jy [3], accounting for the fact that at $8.9 \mathrm{GHz}$ the solid angle of the GBT beam covers only $25 \%$ of the area occupied by the nebula. Some of our sessions were heavily contaminated with broad RFI pulses with typical $\mathrm{S} / \mathrm{N}<10$. Therefore, for further analysis we kept only events with peak flux density exceeding $F_{t h r}=8.1 \mathrm{Jy}(10 \sigma$ for the session with smallest sampling time) and which arrived in the MP or IP phase windows. Additionally, we excluded all events with the width larger than 30 samples, 
Table 1: Summary of observational parameters and GP/ $\gamma$ outcome for each observing date. The columns from left to right are: observing date; time resolution, $\Delta t$; nominal $1 \sigma$ sensitivity; duration of radio observations, $T_{\text {radio }}$; the observing time, simultaneous with Fermi, $T_{\text {simul }}$; the number of GPs $N_{\mathrm{GPs}}$ detected during radio observations and simultaneously with Fermi; and number of gamma photons, $N_{\gamma}$.

\begin{tabular}{llllllll}
\hline $\begin{array}{l}\text { Date } \\
(2009)\end{array}$ & $\Delta t$ & $1 \sigma$ & $T_{\text {radio }}$ & $T_{\text {simul }}$ & \multicolumn{2}{c}{$N_{\text {GPs }}$} & $N_{\gamma}$ \\
\hline Sep 12 & 2.56 & 0.81 & 87.1 & 28.9 & 139 & 8 & 5 \\
Sep 14 & 3.20 & 0.73 & 165.7 & 62.1 & 4375 & 1834 & 10 \\
Sep 16 & 3.84 & 0.66 & 99.3 & 30.6 & 98 & 27 & 4 \\
Sep 19 & 3.20 & 0.73 & 118.5 & 54.3 & 6957 & 1830 & 5 \\
Sep 20 & 3.20 & 0.73 & 110.5 & 32.2 & 1846 & 384 & 2 \\
Sep 21 & 3.84 & 0.66 & 55.1 & 31.5 & 27 & 16 & 2 \\
Sep 22 & 3.20 & 0.73 & 147.7 & 68.9 & 1256 & 603 & 5 \\
Sep 23 & 3.20 & 0.73 & 164.5 & 82.0 & 10520 & 5078 & 10 \\
Sep 24 & 3.84 & 0.66 & 55.3 & 22.5 & 38 & 37 & 1 \\
Sep 25 & 3.20 & 0.73 & 236.5 & 130.8 & 14320 & 10014 & 13 \\
Sep 28 & 3.20 & 0.73 & 72.3 & 48.1 & 34 & 9 & 5 \\
Oct 25 & 3.20 & 0.73 & 157.6 & 41.3 & 3164 & 1261 & 15 \\
\hline Total & & & 1470.0 & 633.1 & 42774 & 21092 & 77
\end{tabular}

as being presumably caused by RFI. This resulted in the selection of more than 40000 GPs (see Table 1). Comparing the number of single pulses above $8.1 \mathrm{Jy}$ and narrower than 30 samples in and out of phase window, we can estimate the fraction of false GPs in our final data set to be less than $0.001 \%$.

For each radio observation session we extracted "Diffuse" class events with energies $>100 \mathrm{MeV}$. Photons with zenith angles greater than $105^{\circ}$ were excluded to eliminate the Earth's bright $\gamma$-ray albedo. We selected only photons in Good Time Intervals (GTIs) within an angle $\theta<\operatorname{Max}(6.68-$ $1.76 \lg (E / 1000 \mathrm{MeV}), 1.3)^{\circ}$ of the radio pulsar position [1]. These photons were barycentered and assigned phases with the TEMPO2 fermi plugin. The timing accuracy of the Fermi/LAT is better than $1 \mu \mathrm{s}$ [1]. Over the course of all radio observations we accumulated 10.5 hours of Fermi data within GTIs, resulting in 77 photons with energies above $100 \mathrm{MeV}$, together with background (see Table 1). The distribution of number and energy/peak intensity of photons/GPs with respect to pulsar rotational phase is shown on Fig 1 . As was reported earlier, $\gamma$-ray and radio emission windows are aligned and we consider GPs come only within MP/IP emission windows.

At high frequencies GPs are strongly affected by interstellar scintillations [3], which change the apparent rate and peak intensity of GPs. Following [11] and [3] we estimated refractive (RISS) and diffractive (DISS) time scales for our observation setup to be about 80 and 9 minutes. The RISS time scale roughly matches the observed day-to-day variation of the GP rate (see Table 1). Within each observing subsession, there appeared to be two short GP rate variations time scales 20 and 2-4 minutes. Both of them agree fairly well with the DISS estimations, considering all the uncertainty in the scintillation parameters. However, on the other side, there is no evidence against 

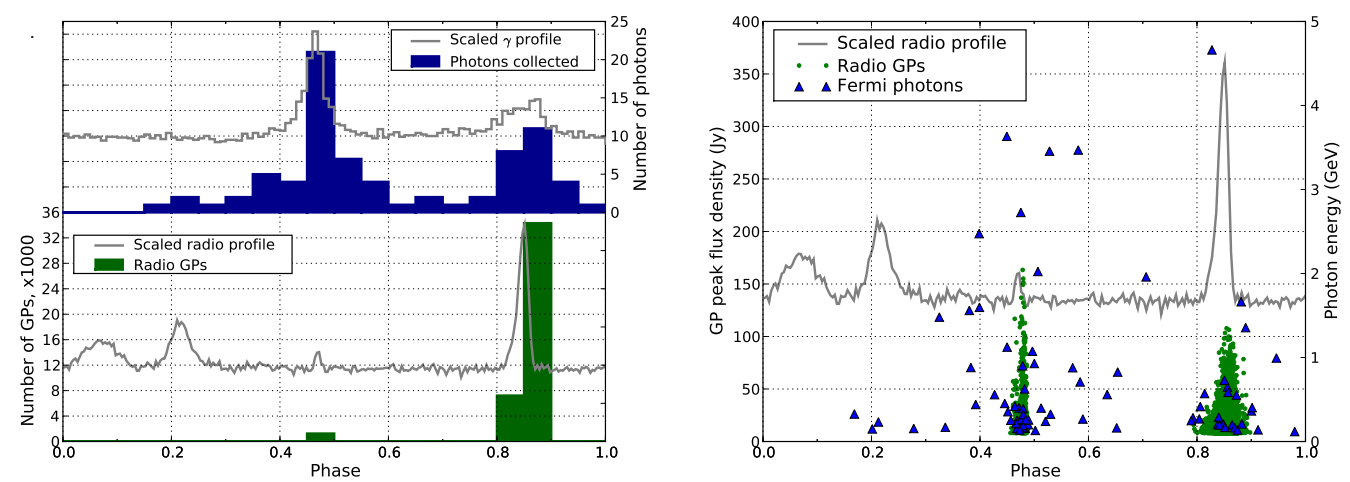

Figure 1: Left: histograms of GPs for all radio observing time (bottom) and Fermi photons during the simultaneous time (top). For illustrative purposes, scaled and shifted along y-axis radio and $\gamma$-ray profiles are shown (gray). Scaled radio profile is from GBT session on Sep 25, 2009, and $\gamma$-ray profile is Fermi profile accumulated during Sep-Oct, 2009. Right: distribution of peak flux density of GPs and energy of $\gamma$-ray photons over pulsar rotational phase. Scaled and shifted along y-axis radio profile from Sep 25 is added.

the hypothesis that at least one of these time scales is due to intrinsic GP rate variability. If observed day-to-day variation of the GP rate and mean intensity is caused by RISS, then one can make a GP sample, corrected for refractive scintillation. To do that we estimated the amount of RISS intensity damping with respect to the session with highest GP rate, then corrected pulse intensities by that amount, and finally excluded all GPs below some common threshold for all sessions. The amount of damping can be assessed by comparing the peak flux density distributions of GPs (see details in Bilous et al. 2010 [2]). Following this procedure, from our dataset we chose 180 intrinsically brightest pulses with TOAs within the Fermi observing time. All GPs would have peak flux density higher than $69 \mathrm{Jy}$, if they were detected during the session with highest GP rate.

\section{Correlation analysis}

Our correlation analysis consisted of two distinct approaches. The first one was to test if GPs tend to cluster, or come in bursts around $\gamma$-ray photons (or to appear in clusters, but with some time lag with respect to the $\gamma$-ray photons). For the second, we wanted to verify the hypothesis that the $\gamma$ ray photon flux increases during GPs. For both cases, the statistical test of significance was done by analyzing the simulated high-energy datasets, for which there was no intrinsic correlation between photons and GPs. The simulation was done with gtobssim software in the Fermi tools package. We used the Pass6_v3 instrument response function, together with the same spacecraft/pulsar ephemeris as in real data analysis. The pulsar was simulated using PulsarSpectrum library, with the light curve, spectrum and integral flux above $100 \mathrm{MeV}$ taken from [1]. The integral flux was set to $F_{\mathrm{av}}=2.09 \times 10^{-6} \mathrm{~cm}^{-2} \mathrm{~s}^{-1}$ for the burst correlation analysis and varied from 0 up to about a hundred $F_{\text {av }}$ for the single-pulse correlation analysis. The Crab nebula was modeled as a point source (for all our energy range its angular diameter is less than the region of interest), with spectrum from [1] and integral flux above $100 \mathrm{MeV}$ of $9.8 \times 10^{-7} \mathrm{~cm}^{-2} \mathrm{~s}^{-1}$. For Galactic and ex- 
tragalactic background we used "GalacticDiffuse_v02" and "IsotropicDiffuse_v02" models. The simulated photon files were processed in the same way as the real data.

To test if occurrence of $\gamma$-ray photons is correlated with the change in GP generation rate, we calculated the distribution of time lags between each photon and all GPs in that photon's GTI. Changing the bin size of the distribution makes it sensitive to different sizes of supposed GP clusters. In this study, we tried a set of bin widths, starting from 10 seconds and increasing the width by 10 seconds up to 2 minutes. Two minutes correspond to the minimum RISS time scale, and on time scales less than $10 \mathrm{~s}$ the Poisson noise due to discrete number of Fermi photons becomes too high. We did 1000 simulation runs and contrasted the real-data distribution with the mean and $95 \%$ percentile of all of the simulated datasets. Figure 2 (left) shows the distribution of time lags between GPs and photons for all GPs for one particular bin width, namely 2 minutes. The real data points lie all within $95 \%$ percentile of simulation, indicating no apparent change in GP generation rate on 2-min time scale with any possible time lag within \pm 40 minutes with respect to the $\gamma$-ray photons. All other bin widths give the same result, for both all GPs and the GP sample corrected for the refractive scintillation.

Another question of interest is whether the average $\gamma$-ray flux from the Crab pulsar increases during individual GPs. To investigate that, we looked for the number of $\gamma$-ray photons in 30-ms window around each GP. For 10.5 hours of simultaneous observations, we detected only one photon within $30 \mathrm{~ms}$ from GP. These photon and radio GP were close to corresponding energy thresholds. Nonetheless, knowing the observed number of matches $N=1$, the probability that the $\gamma$-ray flux during GPs is equal to some value $F_{0}$ can be estimated with simple Bayesian formula:

$$
p\left(F=F_{0} \mid N\right)=\frac{p\left(F_{0}\right) \cdot p\left(N \mid F=F_{0}\right)}{\int_{0}^{F_{\max }} p\left(F_{0}\right) \cdot p\left(N \mid F=F_{0}\right) d F_{0}},
$$

where $p\left(F_{0}\right)$ is the prior distribution for $F_{0}$ and $p\left(N \mid F=F_{0}\right)$ is the likelihood - probability that we would get the observed number of matches $N$ if the pulsar flux during GPs is equal to $F_{0}$. Since little is known about $p\left(F_{0}\right)$, we chose the prior to be uniform in a flux range from 0 (the Crab pulsar turns off $\gamma$-ray emission during GPs) to $F_{\max } \sim 30 F_{\mathrm{av}}$, which corresponded to hypothesis that all photons from the Crab pulsar come during GPs. The likelihood $p\left(N \mid F=F_{0}\right)$ was calculated by running simulations with different pulsed flux $F_{0}$ and computing the fraction of runs with a number of matches $N$. Here we implicitly assumed that a higher flux outside selected windows does not influence the correlation within the windows.

On Fig. 2 (right) we show the resulting probabilities that the $\gamma$-ray flux from the Crab pulsar during GPs does not exceed the given number of times the mean flux reported by [1]. Errors due to the limited number of simulation runs are plotted as error bars, whereas those due to discrete number of matches are given by separate posterior probability curves for $N=0$ and $N=2$.

With 95\% probability, the high energy flux of the Crab pulsar during GPs is less than 4 times average pulsed flux for windows of $30 \mathrm{~ms}$. If to consider the uncertainty due to discrete number of matches between photons and GPs, the 95\% upper limits of the $\gamma$ flux are within 3-5.5 times the average pulsed flux. For more results and details see the Bilous et al. (2010) paper [2].

\section{Acknowledgments}

The National Radio Astronomy Observatory is a facility of the National Science Foundation 

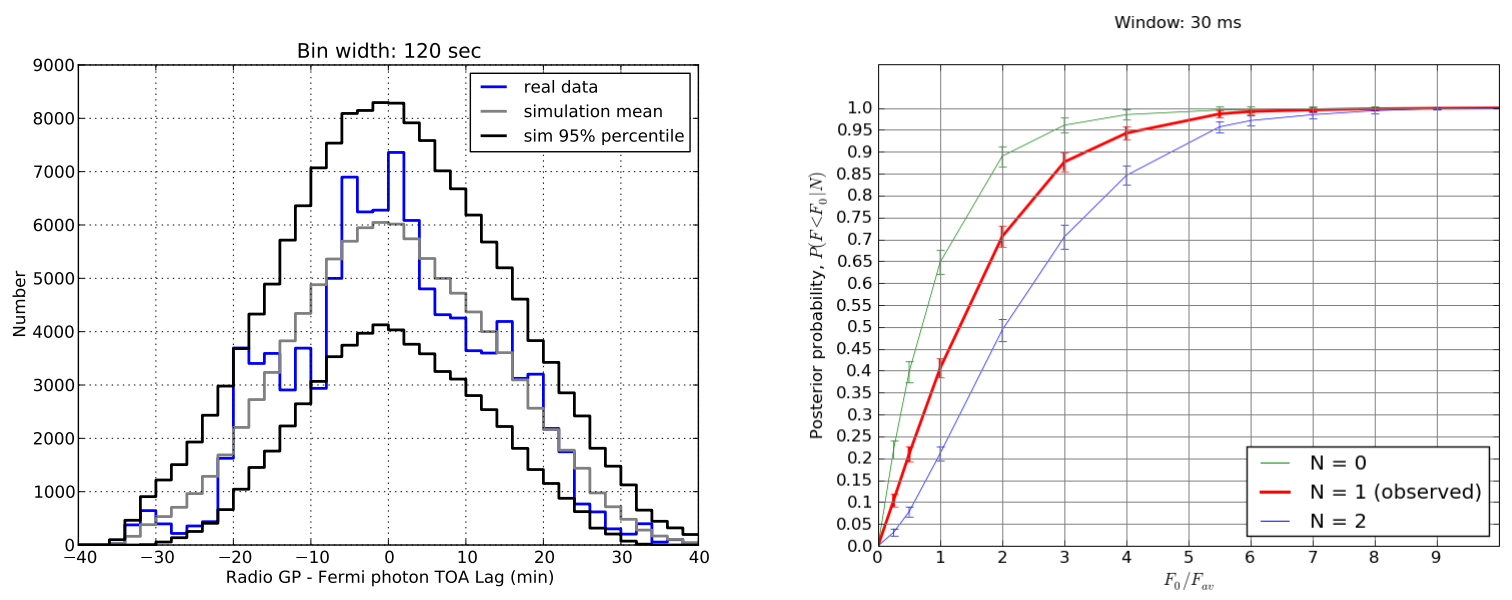

Figure 2: Left: distribution of time lags between GPs and $\gamma$-ray photons for 2-min bins, for all GPs $>8.1 \mathrm{Jy}$. Real Fermi photons (blue line) are contrasted to the mean and $95 \%$ percentile on the pool of simulated datasets (grey and black). The fact that distribution for the real data lies within $95 \%$ of simulated ones indicates no apparent change in GP generation rate on 2-min time scale with any possible time lag within \pm 40 minutes with respect to the $\gamma$-ray photons. All other bin widths (down to $10 \mathrm{~s}$ ) produce the same result. Right: posterior probability that $\gamma$ flux in a $30 \mathrm{~ms}$ window around GP is less than $F_{0}$, knowing the observed number of matches $N=1$. Errors due to the limited number of simulation runs are plotted as error bars, whereas those due to discrete number of matches are given by separate posterior probability curves for $N=0$ and $N=2 . F_{\text {av }}$ - average pulsed $\gamma$-flux from Crab pulsar, reported by [1].

operated under cooperative agreement by Associated Universities, Inc. This work was supported by Fermi grant NNX10AD14G. MAM is supported by a Cottrell Fellowship, a Sloan Fellowship, a WVEPSCOR Research Challenge Grant.

\section{References}

[1] Abdo, A. A. et al. 2010, ApJ, 708, 1254

[2] Bilous, A. V., Kondratiev, V. I., McLaughlin, M. A., et al. 2010, ApJ, submitted

[3] Cordes, J. M., Bhat, N. D. R., Hankins, T. H., McLaughlin, M. A., \& Kern, J. 2004, ApJ, 612, 375

[4] Knight, H. S., Bailes, M., Manchester, R. N., Ord, S. M., \& Jacoby, B. A. 2006, ApJ, 640, 941

[5] Lundgren, S. C. 1994, PhD thesis, Cornell Univ., Ithaca, NY

[6] Lundgren, S. C., Cordes, J. M., Ulmer, M., et al. 1995, ApJ, 453, 433

[7] Moffett, D. A. 1997, PhD thesis, New Mexico Inst. Mining and Technology

[8] Moffett, D. A., \& Hankins, T. H. 1996, ApJ, 468, 779

[9] Popov, M. V., \& Stappers, B. 2007, A\&A, 470, 1003

[10] Ramanamurthy, P. V., \& Thompson, D. J. 1998, ApJ, 496, 863

[11] Rickett, B. J., \& Lyne, A. G. 1990, MNRAS, 244, 68

[12] Shearer, A., Stappers, B., O’Connor, P., et al. 2003, Science, 301, 493 